CVIA

REVIEW ARTICLE

pISSN 2508-707X / eISSN 2508-7088 https://doi.org/10.22468/cvia.2017.00031 CVIA 2017;1(3):166-176

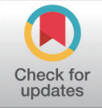

Received: February 28, 2017

Revised: June 16, 2017

Accepted: June 16, 2017

Corresponding author

Jin Hur, MD, PhD

Department of Radiology,

Severance Hospital, Yonsei University

College of Medicine, 50 Yonsei-ro,

Seodaemun-gu, Seoul 03722, Korea

Tel: 82-2-2228-7400

Fax: 82-2-393-3035

E-mail: khuhz@yuhs.ac

\section{Technological Improvements} in Cardiac Thrombus Diagnosis

\author{
Jin Hur, Yoo Jin Hong, Dong Jin Im, Hye-Jeong Lee, \\ Young Jin Kim, Byoung Wook Choi, \\ Department of Radiology, Severance Hospital, Yonsei University College of Medicine, Seoul, Korea
}

\begin{abstract}
Cardiovascular imaging technology constantly evolves and provides more testing options for evaluating cardiovascular morphology and function. Accurate detection and diagnosis of cardiac thrombi are important in clinical practice, as they provide a substrate for embolic events and a rationale for anticoagulation therapy. Echocardiography, predominantly transesophageal echocardiography, has been used as a reference modality to assess flow stasis and cardiac thrombus. Cardiac computed tomography (CCT) and cardiac magnetic resonance imaging (CMRI) have also been proposed and tested as alternative imaging modalities for cardiac thrombus detection. Given the noninvasive nature of CCT and CMR, developing strategies based on these modalities for the detection of cardiac thrombi and flow stasis is of great clinical interest. However, these advanced imaging techniques should add value in the clinical setting.
\end{abstract}

Key words Echocardiography - Computed tomography - Magnetic resonance imaging Heart · Thrombus.

\section{INTRODUCTION}

Visualization of anatomic and pathological conditions within the heart, using noninvasive means, is fundamental for understanding, diagnosing, and treating cardiovascular diseases. Cardiovascular system imaging is technically challenging; it is not surprising that cardiovascular applications have often been a driving force in the ongoing evolution of imaging technologies.

Atrial fibrillation (AF) is a major independent risk factor for stroke, and the risk increases in patients with $\mathrm{AF}$ and other concomitant potential cardiac sources of embolism [1-4]. Thus, accurate detection and diagnosis of high-risk embolic sources, such as cardiac thrombi, is important, as they provide a substrate for embolic events and a rationale for anticoagulation therapy. For almost 2 decades, the gold standard for assessing flow stasis and thrombi in the heart has undoubtedly been echocardiography, predominantly transesophageal echocardiography (TEE) [5-7]. Intra-cardiac echocardiography (ICE) and TEE evolved from transthoracic echocardiography (TTE) as an approach that uses high-frequency transducers with improved spatial resolution. Cardiac computed tomography (CCT) and cardiac magnetic resonance imaging (CMRI) have also been proposed and tested as alternative imaging modalities for cardiac thrombus

(c) This is an Open Access article distributed under the terms of the Creative Commons Attribution Non-Commercial License (http://creativecommons.org/licenses/by$\mathrm{nc} / 4.0$ ) which permits unrestricted non-commercial use, distribution, and reproduction in any medium, provided the original work is properly cited. detection [8-13]. More recently, new imaging techniques, such as dual-energy CT (DECT), have been developed and tested to assess flow stasis and cardiac thrombi [14].

In this article, we describe the pathophysiology of thrombus formation. We introduce recent technical advances in cardiovascular imaging in the fields of echocardiography, CT, and MRI as approaches to evaluate flow stasis and cardiac thrombus. We also discuss advantages and disadvantages of the available imaging modalities for cardiac thrombus assessment.

\section{CARDIAC THROMBUS}

\section{LAA function and thrombus formation}

Anatomically, the left atrial appendage (LAA) is highly variable, and consists of a blind-ended pouch with tapering edges and trabeculations from the pectinate muscles. This morphology promotes stasis and thrombus formation. The shape of the LAA can correlate with different risks of thrombus formation and stroke, although this remains controversial; a few studies have shown that extensive trabeculations are associated with stroke $[15,16]$.

Normal contraction of the LAA during sinus rhythm and adequate blood flow within the LAA lower the risk for thrombus formation within the cavity. Thrombus formation is more likely to occur within the LAA in cases with reduced contractility and stasis. During AF, there is a decrease in LAA contrac- 
tility and function, which manifests as a decrease in Doppler velocities and LAA dilatation [17]. The remodeling processes associated with AF causes the LAA to function as a static pouch, predisposing the heart to stagnation and thrombosis. AF results in structural remodeling changes in the LAA, especially chamber enlargement. The average LAA volume is more than 3 times larger in $\mathrm{AF}$ patients, compared with patients in sinus rhythm [18]. Other structural LAA changes those in AF include luminal surface area enlargement, a smoother endocardial surface, and a higher degree of endocardial fibroelastosis, all of which can all contribute to thrombus formation $[18,19]$.

\section{LA or LAA thrombus}

The left atrium (LA) and the LAA are important locations that are frequently associated with thrombus formation and subsequent cardioembolic events, particularly in association with dysrhythmias, such as AF [20,21]. An estimated $47 \%$ of thrombi in valvular $\mathrm{AF}$ and $91 \%$ of those in nonvalvular $\mathrm{AF}$ are located in the LAA [22]. Therefore, current research is focused on the LAA to better understand its anatomy, physiology, and to test various imaging modalities and techniques to assess the shape and size, blood flow patterns, and for the presence or absence of thrombi. Cardiac thrombi of the LA or LAA are common causes of stroke, and because the LA and LAA thrombi are treatable sources of embolism, thrombi detection may significantly affect patient management.

\section{LV thrombus}

Left ventricular (LV) thrombi can occur in LV dysfunction, especially in the acute stage after myocardial infarction (MI)
$[23,24]$. LV thrombi are clinically important because of their ability to embolize $[25,26]$. In fact, patients who develop mural thrombi after MI have a poor prognosis, including a $10 \%$ rate of systemic embolization [27]. Early detection of LV thrombi is critical because it allows for early initiation of anticoagulation therapy to reduce the likelihood of embolization [28].

\section{Right heart thrombus}

Right heart thrombus (RHT) in the absence of structural heart disease, atrial fibrillation, or a cardiac catheter is rare. The majority of thrombi originates from the deep venous system and embolize to the right heart as precursors of a pulmonary embolism (PE) [29]. Clinical consequences of RHT depend upon the clot size and overall clot burden. Thrombi in the right side of the heart may become infected or cause a PE. Sudden cardiovascular collapse is the worst possible outcome if the clot compromises the cardiac or pulmonary circulation. The overall mortality rate in patients with RHT has been reported as $28 \%$ and as high as $100 \%$ in untreated patients [30,31]. It is important to diagnose an RHT, because it can embolize at any moment, which would then require emergency treatment, and this type of embolism is associated with a high mortality rate.

\section{IMAGING MODALITIES}

\section{Echocardiography}

Initial studies that use TTE have a limited ability for detecting LA and LAA thrombus formation [7,32]. However, harmonic imaging and the administration of ultrasound contrast agents have enhanced the ability of TTE to detect LAA thrombi [33,34].
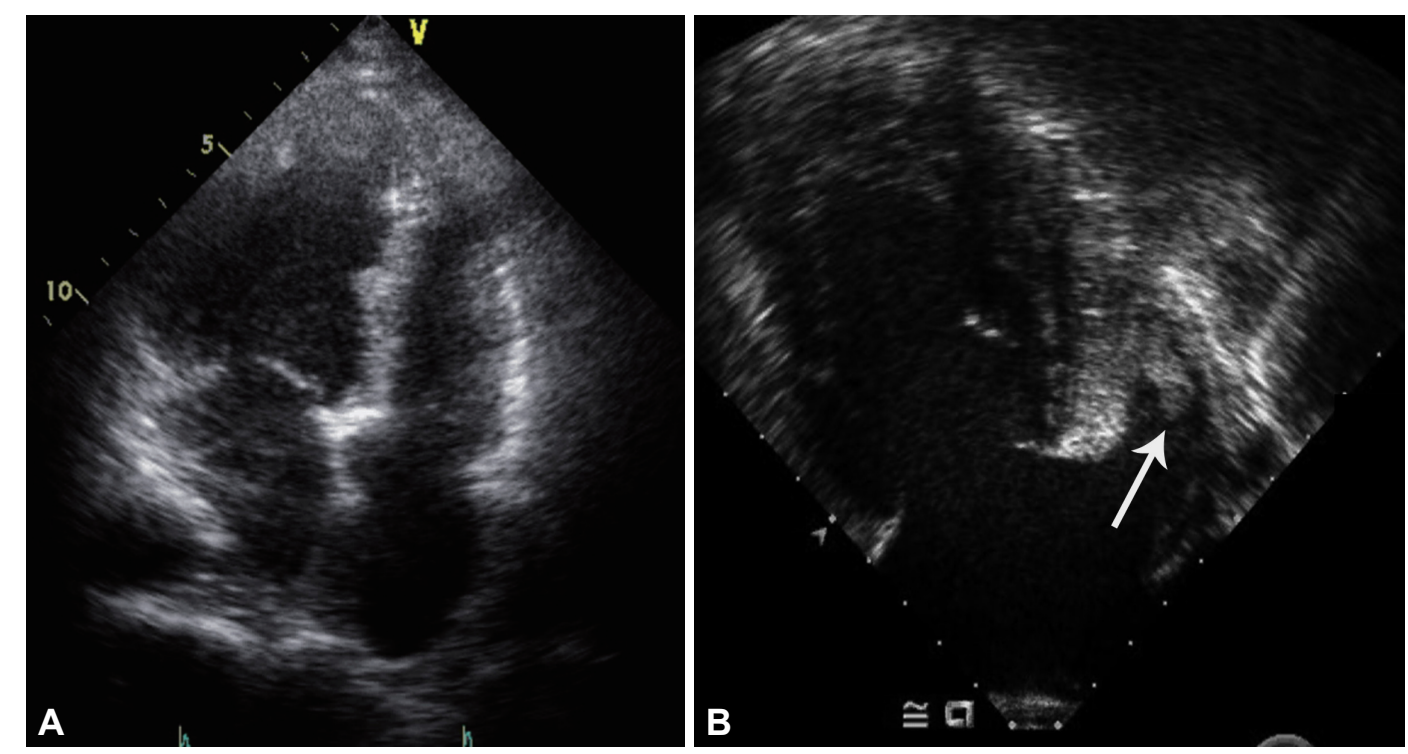

Fig. 1. Images from a 45-year-old man with stroke and atrial fibrillation. (A) 4-chamber TTE image shows no thrombus in the LA. (B) TEE image reveals a small echogenic thrombus (arrow) in the LAA. LA: left atrium, LAA: left atrial appendage, TEE: transesophageal echocardiography, TTE: transthoracic echocardiography. 
Because of the superior visualization of posterior structures, such as the LA and LAA, compared with TTE, TEE has been shown to be effective for assessing thrombi in the LA and LAA (Fig. 1) [35,36]. Therefore, TEE is currently the most widelyused method and is accepted as the reference modality for diagnosing and excluding the presence of LAA thrombi. However, TEE is a semi-invasive procedure that requires special skills for proper execution and interpretation. A prospective study reported $100 \%$ sensitivity [ $95 \%$ confidence interval (CI): 74-100\%], 99\% specificity (95\% CI: 97-99.9\%), and 100\% negative predictive value (NPV) (217/217), but only an $86 \%$ positive predictive value (PPV) (12/14) for a population with a 5.2\% thrombi prevalence [36]. The investigators suggested that this low PPV resulted from trabeculations or pectinate muscles in the LAA being misinterpreted as thrombi by inexperienced observers.

One drawback of TEE is that it has limited sensitivity for identifying small thrombi or thrombi within a side lobe. Thus, the absence of LAA thrombus visualization does not always equate with the absence of an LAA thrombus. Functional assessment of the LAA using Doppler echocardiography is widely-used to better assess the LAA and the risk of thromboembolism. LAA blood flow velocity measurement allows a more-quantifiable assessment of spontaneous echo contrast (SEC) than grades of severity, which are primarily based on echogenicity. Decreasing LAA velocities were strongly associated with increasing grades of SEC severity in the LA and LAA, and with embolic events [37].

The use of three-dimensional (3D) TEE imaging is a relatively recent development that improves assessment of LAA anatomy. Although two-dimensional (2D) TEE provides higher-resolution images, because of a better frame rate, 3D TEE allows a more comprehensive assessment of the LAA and overcomes some of the limitations associated with 2D imaging, such as in- adequate imaging planes. In addition, 3D TEE provides better separation and differentiation between adjacent structures, along with a more complete and comprehensive evaluation of the LAA, its complex morphology, and the surrounding structures [38]. There are still limited data regarding the sensitivity and specificity of 3D TEE for detecting LAA thrombi. However, 3D TEE has become an important tool for guiding therapeutic devices into the LAA with recent advances in percutaneous devices for LAA closure.

ICE can be used as an alternative imaging method to TEE to assess LAA flow stasis and thrombus formation. ICE can provide multiple views and detailed LAA imaging which facilitates detection of potential thrombi $[39,40]$. Although ICE is less sensitive than TEE for thrombus detection [41], it can serve as a complementary method, especially when equivocal TEE findings merit further evaluation. However, because ICE is an invasive procedure, it is not typically used in daily practice and is mainly reserved for planned interventional cardiac procedures in a catheterization laboratory setting.

2D TTE was the first imaging modality for visualizing LV thrombi [42]. 2D TTE has been reported to have sensitivity rates of $92 \%$ to $95 \%$ and specificity rates of $86 \%$ to $88 \%[43,44]$. Despite its high diagnostic accuracy, TTE can be technically challenging, as echocardiographic findings of the LV thrombus may be subtle and, consequently, easily missed. The disadvantages of TTE include difficulty in discerning the myocardium-thrombus interface, limited near-field resolution of the apex, and difficulty in obtaining images of the true apex. As a result, small non-protruding mural thrombi may resemble a normal or mildly thickened LV wall, and normal structures, such as papillary muscles, anomalous bands, and trabeculae may mimic thrombi. TEE has been shown to be better suited than TTE for detecting intra-atrial
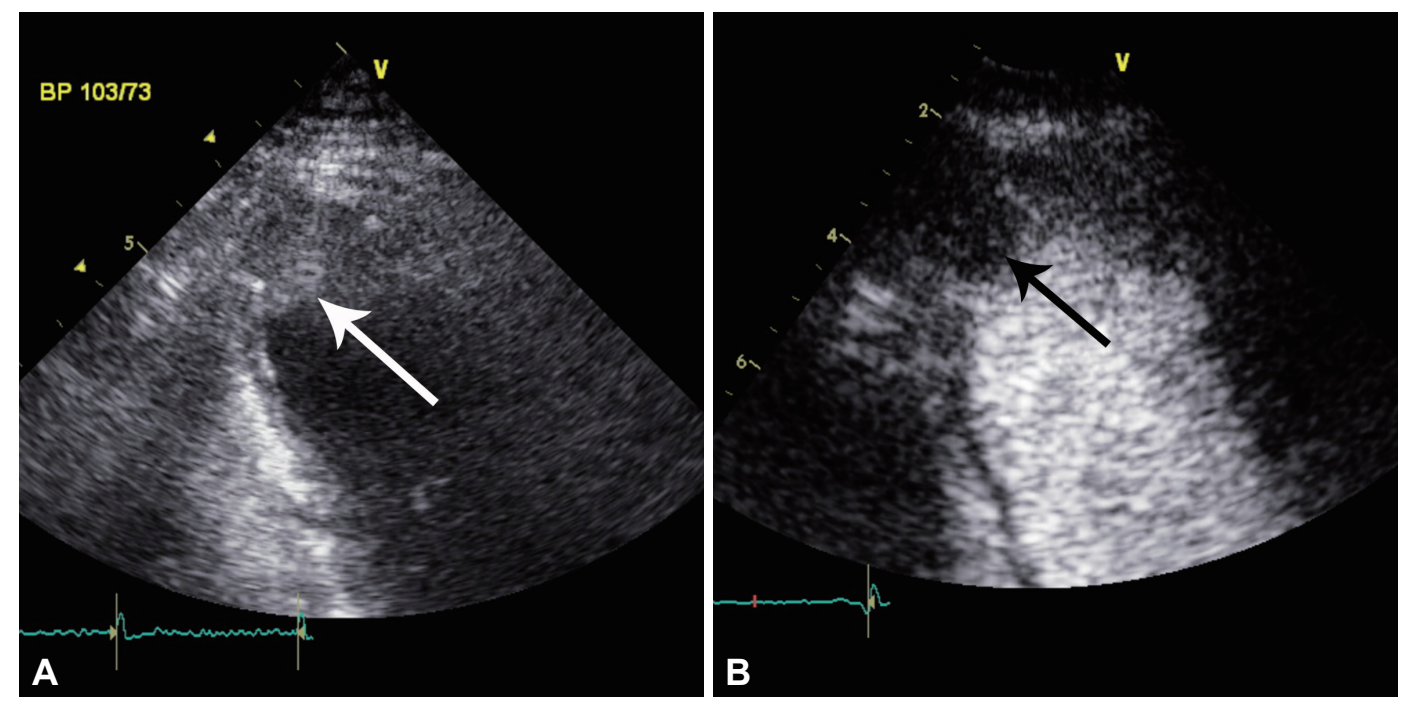

Fig. 2. Image from a 68-year-old man with stroke and myocardial infarction. (A) TTE image shows a suspicious echogenic thrombus (arrow) in the LV apex. The thrombus evaluation was difficult because of poor acoustic windows. (B) TTE with contrast agent shows a small thrombus (arrow) in the LV apex. TTE: transthoracic echocardiography, LV: left ventricle. 
thrombi, but its value in the diagnosis of LV thrombus has not been extensively explored $[45,46]$. A previous study has shown that when TTE and TEE are used to detect or exclude the LV thrombus, the results may be inconclusive in as many as $46 \%$ (87/190) of patients [47]. However, advanced techniques, such as harmonic imaging and the use of intravenous echocardiographic contrast agents, may improve the overall diagnostic image quality [48]. Thus, LV echocardiographic contrast agents have been used to increase the accuracy of LV thrombus detection, especially in patients with difficult acoustic windows (Fig. 2).

TTE is usually considered as a screening test for diagnosing right heart thrombi. TTE may demonstrate right ventricular dysfunction, tricuspid regurgitation, and leftward atrial septal bowing in patients with pulmonary thromboemboli. However, direct visualization of the thrombus located in the pulmonary arteries or entrapped in the right atrium is difficult. In contrast, TEE not only detects the thrombus in the heart with higher accuracy, it may also allow $\mathrm{PE}$ to be diagnosed, and is associated with $80 \%$ sensitivity and $100 \%$ specificity in patients with suspected massive pulmonary emboli [49]. TEE can also provide information about the presence of a right-to-left shunt through a patent foramen ovale.

\section{Cardiac computed tomography}

A non-invasive method for identifying intracardiac thrombus with high reliability and accuracy, comparable to TEE, would be of significant clinical value. CCT is a well-established, but not widely-used technique for cardiac thrombus imaging. CCT can be used to detect intracardiac thrombus with high diagnostic accuracy (Fig. 3) [8-11,50,51]. A recent meta-analysis demonstrated that the overall high accuracy of cardiac CT, compared with TEE, can be used to detect LA or LAA thrombus in patients with AF. The investigators included 19 studies with a total of 2955 patients and found the weighted mean sensitivity and specificity to be 96\% (95\% CI: 92-100\%) and 92\% (95\% CI: 91-93\%), whereas the PPV and NPV were $41 \%$ (95\% CI: 37-44\%) and 99\% (95\% CI: $99-100 \%)$, respectively [52]. This is because a pseudo-filling defect, such as flow stasis, can also cause an apparent filling defect on CT images, thereby mimicking a thrombus. Because CCT identifies a cardiac thrombus based on anatomic appearance, it can be challenging to differentiate between thrombus and flow stasis.

The flow stasis phenomenon appears when LA dysfunction causes incomplete mixing of contrast agent and blood. Therefore, delayed phase scanning would allow for complete mixing of contrast agent and blood (Figs. 4 and 5) [53,54]. In a subanalysis of 753 patients from a recent meta-analysis with available delayed imaging data, the PPV increased significantly to $92 \%$, while maintaining a high NPV of $100 \%$ and an overall diagnostic accuracy of 99\% (95\% CI: 98-100\%) [52]. These results suggest that cardiac CT using delayed imaging is a reasonable alternative to TEE to evaluate LAA or LA thrombus and to differentiate thrombus from flow stasis.

Although delayed imaging with cardiac CT has excellent sensitivity and specificity for thrombus, it requires rescanning within 1 minute. Since this would double the radiation exposure, prior knowledge of or a high suspicion of LA or LAA thrombus would be required before patients would be subjected to this procedure. Therefore, radiation exposure is a significant disadvantage of cardiac CT for LAA evaluation. However, CT has recent-
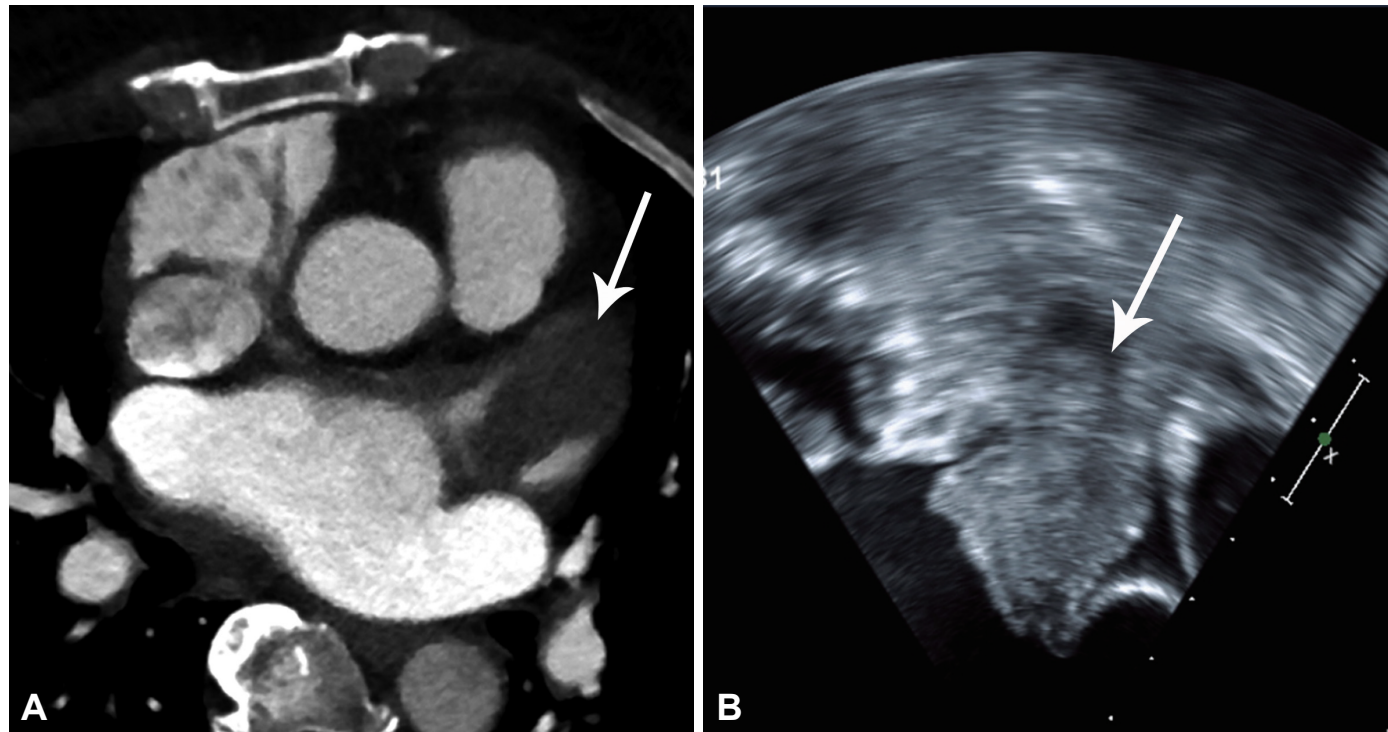

Fig. 3. Images from a 62-year-old woman with atrial fibrillation. (A) Axial cardiac CT imaging shows an oval filling defect (arrow) in the LAA. (B) TEE imaging demonstrates an oval-shaped echogenic thrombus (arrow) in the LAA. LAA: left atrial appendage, TEE: transesophageal echocardiography. 


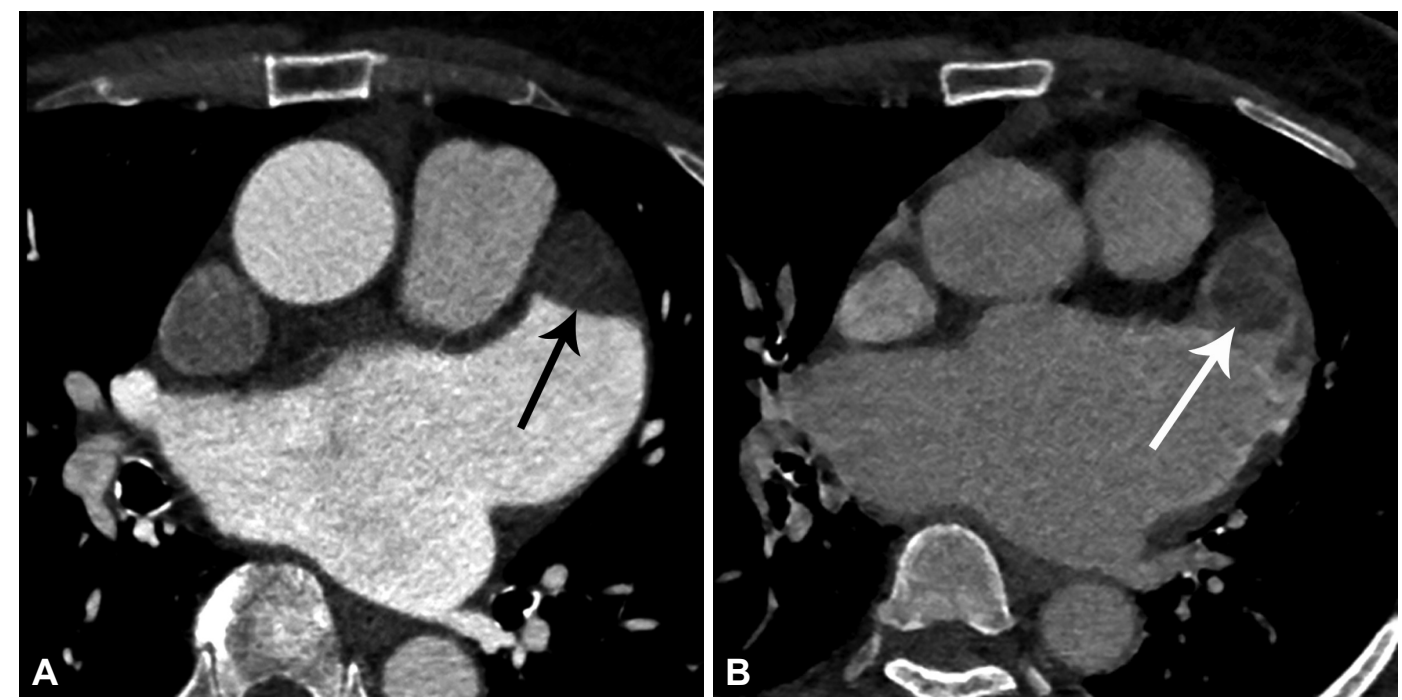

Fig. 4. Images from a 65-year-old woman with stroke and atrial fibrillation. (A) Axial early-phase cardiac CT imaging shows a triangular filling defect (arrow) in the LAA. (B) Axial late-phase cardiac CT imaging shows a round-shaped thrombus (arrow) in the LAA. LAA: left atrial appendage.


Fig. 5. Images from a 41-year-old man with stroke and atrial fibrillation. (A) Axial early-phase cardiac CT image displays a triangular filling defect (arrow) in the LAA. (B) Axial late-phase cardiac CT image shows no filling defect (arrow) in the LAA. The defect caused by blood stasis disappeared during late-phase imaging. (C) TEE image demonstrates moderate spontaneous echo contrast (arrow) with no thrombus in the LAA. LAA: left atrial appendage, TEE: transesophageal echocardiography.

ly undergone technical and acquisition changes that substantially reduce the dose of radiation [55].

Recent advancement in CT technology enabled DECT to generate material-specific images based on the atomic number $\mathrm{Z}$ and the unique mass attenuation coefficient of a particular material at different X-ray energies [56,57]. Material-specific images provide qualitative and quantitative information about tissue composition and contrast media distribution. The most significant contribution of DECT-based material characterization comes from the capability to assess iodine distribution by creating an image that exclusively shows iodine. These iodine-specific images increase tissue contrast and amplify subtle attenuation differences between normal and abnormal tissues, improving lesion detection and characterization. The DECT technique permits the differentiation of iodine from other materials by the material decomposition method [58]. This is valuable for differentiating iodine-enhancing lesions, such as flow stasis or tumors, from non-enhancing lesions including thrombus (Figs. 6 and 7). Early experiences with dual-energy CCT for detecting thrombi and blood flow stasis in 63 stroke patients demonstrated that the overall sensitivity and specificity were $97 \%$ (95\% CI: $82-100 \%)$ and $100 \%$ (95\% CI: 86-100\%), respectively. In addition, the PPV was $100 \%$, while maintaining a high NPV of $97 \%$ [14]. This result suggests that DECT has the potential to detect LAA thrombus and distinguish thrombus from flow stasis. Another study of dual-energy CCT in 37 patients with cardiac masses demonstrated that the iodine concentration could be feasibly quantified from DECT data and used to differentiate cardiac myxomas from thrombi. Based on these results, the quantified values of the mean iodine concentration $(\mathrm{mg} / \mathrm{mL})$ were significantly higher in cardiac myxomas compared to cardiac thrombi $(3.53 \pm 0.72$ vs. $1.37 \pm 0.31$, respectively, $\mathrm{p}<0.001$ ) [59]. This study also supported 



Fig. 6. Images from a 52-year-old man with stroke and thrombus. (A) Axial cardiac CT shows an oval filling detect (arrow) in the LAA. (B) Axial dual-energy CT iodine map reveals a filling defect in the LAA; the mean iodine concentration within the ROI was $0.66 \mathrm{mg} / \mathrm{mL}$. LAA: left atrial appendage, ROI: region of interest.
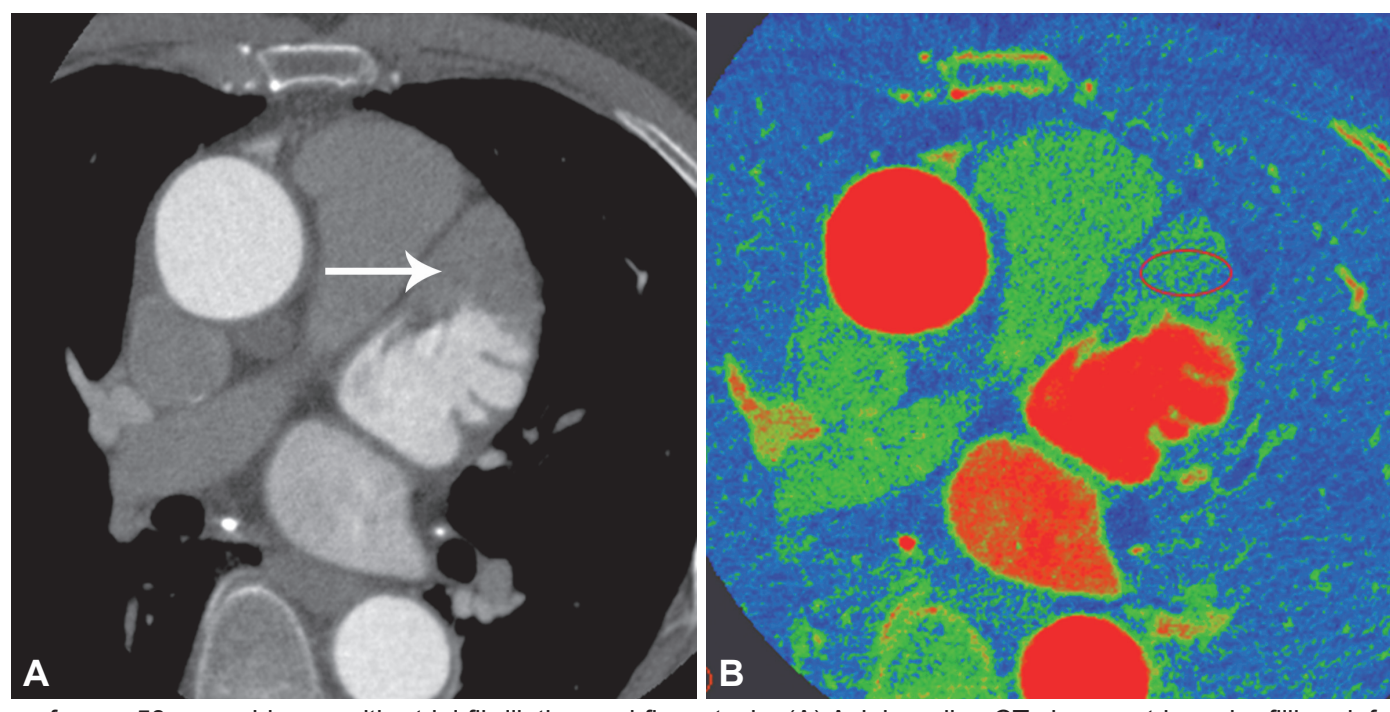

Fig. 7. Images from a 58-year-old man with atrial fibrillation and flow stasis. (A) Axial cardiac CT shows a triangular filling defect (arrow) in the LAA. (B) Axial dual-energy CT iodine map reveals a filling defect in the LAA; the mean iodine concentration within the ROI was $3.36 \mathrm{mg} /$ $\mathrm{mL}$. LAA: left atrial appendage, ROI: region of interest.

that dual-energy CCT using a quantitative analytic methodology can be used to differentiate and characterize iodine-enhancing and non-enhancing lesions.

Prognosis assessment is very important for therapeutic options and approaches in patients with pulmonary thromboembolism. Several studies reported that patients with RHT and PE had worse prognoses than control groups $[60,61]$. The International Cooperative Pulmonary Embolism Registry demonstrated that among patients with acute pulmonary thromboembolism, RHT was a marker of worse prognosis in initially apparently stable patients treated with heparin alone [61]. In this respect, CCT can be used to simultaneously diagnose RHT in patients with acute pulmonary thromboembolism. However, there are not currently any data on contrast media administration or stan- dard scanning protocols.

\section{Cardiac magnetic resonance imaging}

Important advances have been made in rapid MR imaging technology and its application for cardiovascular imaging during the past decade. Clinical high-field strength magnets, highperformance gradient hardware, and ultrafast pulse sequence technology are rapidly improving CMR imaging examination.

CMR imaging can be used to evaluate potential sources of emboli, such as LAA thrombi or LV thrombi [62-64]. Several studies have reported that CMR has high reproducibility and high sensitivity for detecting LAA thrombus, compared to TEE $[62,63]$. Diagnostically, CMR imaging is potentially more advantageous, compared with echocardiography because of its 
ability to characterize the myocardium, in addition to providing cine imaging (cine-CMR). However, one of the drawbacks of CMR is that it is far from an ideal modality as it has limited spatial resolution and susceptibility to slow flow, which can create artifact-mimicking, space-occupying lesions. Spatial resolution and image contrast between the background and LA and LAA wall, as well as slow flow within the LA and LAA, can affect the 3-dimensional contrast-enhanced CMR thrombus evaluation [63]. Imaging artifacts are also commonly encountered in cine-CMR; these include breathing motion, flow-related, and inhomogeneity artifacts.

Recent advances in sequence development, combined with the ability of paramagnetic contrast agents to enhance the ventricular blood pool, provide delayed enhancement (DE)-CMR with a potential advantage for detecting LA or LV thrombi (Fig. 8). DE$\mathrm{CMR}$, is widely-used to discern viable from infarcted myocardia, and a thrombus can be identified by the absence of contrast en- hancement. DE-CMR has been validated as an accurate technique for thrombus detection based on comparisons with pathological findings and clinical embolic events [13,64-66]. Because DE-CMR identifies a thrombus based on tissue characteristics rather than anatomic appearance, it allows a thrombus to be delineated from the myocardium and chamber cavity irrespective of location or morphology. A previous study reported that, among LV thrombi detected in 12 patients that used DE-CMR, $50 \%(6 / 12)$ were not detected by cine-CMR, and 58\% (7/12) were not detected by echocardiography [13]. Another study reported that DE-CMR identified LV thrombi in 7\% of subjects (55 patients), while cine-CMR identified thrombi in only $4.7 \%$ (37 patients). Among the 55 patients with LV thrombi discovered using DE-CMR, 44\% (24/55) had prior cine-CMR analyses that were negative for thrombus [65]. This study validated the ability of long inversion time (TI) DE-CMR to detect LV thrombi and also showed the superiority of this technique over cine-CMR.
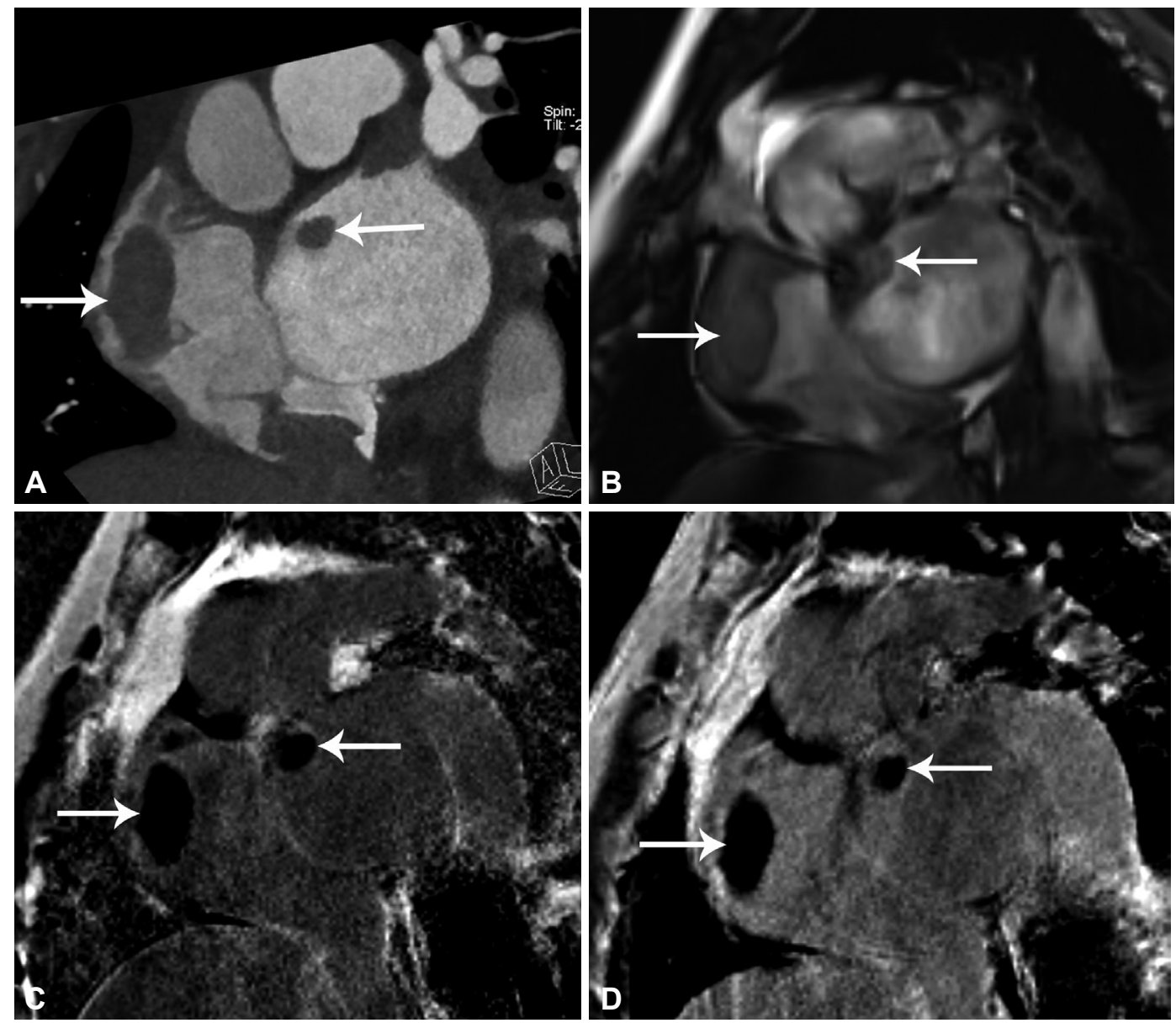

Fig. 8. Images from a 52-year-old woman with atrial fibrillation and dilated cardiomyopathy. (A) Oblique sagittal reformatted CT imaging shows multiple filling defects (arrows) in the RA and LA. (B) A short-axis cine-CMR image, using a balanced steady-state free precession sequence, shows multiple masses (arrows) with iso-signal intensity compared to that of the left ventricular myocardium in the RA and LA. (C) On short-axis standard DE-CMR [inversion time (TI), $220 \mathrm{msec}$ ], the masses showed black signal intensity without delayed enhancement (arrows). (D) On short-axis DE-CMR with long DE-CMR (TI, $400 \mathrm{msec}$ ), the masses showed black signal intensity without delayed enhancement (arrows). The masses were confirmed as thrombi which were resolved on follow up transesophageal echocardiography after anticoagulation therapy. RA: right atrium, LA : left atrium, CMR: cardiac magnetic resonance, DE-CMR: delayed enhancement-CMR. 
Furthermore, the thrombus prevalence increased with worsening LV ejection fraction, ischemia etiology, and myocardial scarring. Therefore, the established capabilities of CMR imaging in coronary artery disease are of additional value when characterizing a suspected thrombus.

A recent meta-analysis compared the diagnostic ability of TTE without contrast, TTE with contrast, cine-CMR, and DE-CMR to detect LV thrombi [67]. The investigators included 7 studies with a total of 803 patients and demonstrated that DE-CMR imaging was the most accurate modality for detecting LV thrombi (sensitivity $88 \%$, specificity $99 \%$ ), followed by cine-CMR imaging (sensitivity 58-79\%, specificity 99\%, accuracy 95\%, PPV 9395\%, NPV 95-96\%), contrast TTE (sensitivity 23-61\%, specificity $96-99 \%$, accuracy 92\%, PPV 93\%, NPV 91\%), and, finally, non-contrast TTE (sensitivity $24-33 \%$, specificity $94-95 \%$, accuracy $82 \%$, PPV 57\%, NPV 85\%). In this meta-analysis, they were not able to demonstrate differential diagnostic accuracy among the different modalities because of the small number of studies and variable usage of gold standards.

A recent study evaluated the diagnostic performance of a comprehensive multicomponent CMR for assessing LA/LAA thrombus in 261 patients with AF that were referred for pulmonary vein isolation [68]. Long TI DE-CMR had the best diagnostic accuracy out of the CMR sequences that were investigated (99.2\%; 95\% CI: 97.2-99.9\%), sensitivity (100\%; 95\% CI: 66.4-100\%), specificity (99.2\%; 95\% CI: 97.2-99.9\%), PPV (81.8\%; 95\% CI: 48.2-97.7\%), and NPV (100\%; 95\% CI: 98.5-100\%). In contrast, cine-CMR had the lowest diagnostic accuracy at $91.6 \%$ (95\% CI: 87.5-94.6\%), sensitivity of $66.7 \%$ (95\% CI: 29.9-92.5\%), specificity of $92.5 \%$ (95\% CI: $88.5-95.4 \%$ ), and a PPV of $24 \%$ (95\% CI: 9.4-45.1\%). All of the CMR sequences had a high negative predictive value, ranging from $98.7 \%$ by cine-CMR to $100 \%$ by long TI DE-CMR. This was likely because long TI DE-CMR provided adequate coverage of the entire LA and LAA and was less susceptible to artifacts. Furthermore, the situation of an under filled LAA could also be avoided, as it was performed with a single-shot technique in the equilibrium phase, $10 \mathrm{~min}$ after contrast administration. In addition, due to the rapid acquisition time of this technique, breath-holding was not required. The processes could also be performed in conditions with irregular cardiac rhythm, which is common in patients with AF.

Myocardial longitudinal relaxation time measurement (T1 mapping) with gadolinium contrast-enhanced inversion recovery-prepared sequences has emerged as a novel approach for noninvasive quantification of myocardial fibrosis. T1 mapping consists of quantifying the T1 relaxation time of a tissue, using analytical expressions of image-based signal intensities. A fundamental principal of MR imaging is that the signal intensity of pixels is based on the relaxation of hydrogen nuclei protons in a static magnetic field $[69,70]$. The T1 relaxation times of two tissues vary substantially. Edema, fat infiltration, and fibrosis also cause differences in $\mathrm{T} 1$ relaxivity. Qualitative sequences rely on the use of arbitrary signal intensity scales for T1 and T2 values with interpatient and inter-image variability, whereas myocardial mapping offers the potential to produce images that have standardized, reproducible scales, similar to the attenuation values used for CT $[71,72]$. Although T1 mapping is currently expanding as a means for depicting diffuse interstitial fibrosis in a variety of cardiac diseases, this technique is rarely used for focal cardiac disease. A few studies have investigated the use of T1 or T2 mapping for evaluating cardiac masses [73,74]. A recent study compared the post-contrast $\mathrm{T} 1$ time derived from $\mathrm{T} 1$ mapping using a TI scout (Lock-Locker sequence) for 15 tumors and 15 thrombi [73]. As expected, the T1 time was significantly shorter in tumors than in thrombi $(383 \pm 84 \mathrm{~ms}$ vs. $477 \pm 139 \mathrm{~ms}, \mathrm{p}=0.03)$. When a cut-off value of $422 \mathrm{~ms}$ was used as a diagnosis of thrombus, the sensitivity and specificity were $67 \%$ and $80 \%$, respectively, with an area under the receiver-operating characteristic curve of 0.73 (95\% CI: 0.54-0.92). Another recent study investigated T1 and $\mathrm{T} 2$ values in 22 patients with cardiac thrombus. According to their data, the native $\mathrm{T} 1$ of thrombi was $1037 \pm 152 \mathrm{~ms}$ (vs. $1032 \pm 39 \mathrm{~ms}$ for myocardium, $\mathrm{p}=0.88$; vs. $1565 \pm 88 \mathrm{~ms}$ for blood pool, $\mathrm{p}<$ 0.0001). T2 were $74 \pm 13$ ms (vs. $51 \pm 3$ ms for myocardium, $\mathrm{p}<$ 0.0001; vs. $170 \pm 32 \mathrm{~ms}$ for blood pool, $\mathrm{p}<0.0001$ ) [74].

CMR imaging is advantageous, compared with cardiac CT, due to the absence of radiation exposure and the avoidance of iodinated contrast media. However, in contrast to CT technology, the future of CMR in assessing cardiac thrombi and flow stasis is less certain. Given the noninvasive nature of CMR and the lack of ionizing radiation, developing strategies based on this modality for detection of cardiac thrombi and flow stasis is of great clinical interest.

\section{CONCLUSION}

The accurate detection and diagnosis of cardiac thrombi are important, as they provide a substrate for embolic events and a rationale for anticoagulation therapy. Substantial efforts have been made to improve the evaluation of flow stasis and thrombi detection. For almost 2 decades, the gold standard for assessing flow stasis and thrombus in the heart has undoubtedly been echocardiography, predominantly TEE. However, a non-invasive method with high reliability and accuracy comparable to TEE that is capable of identifying intra-cardiac thrombus or flow stasis would be of significant clinical value. Currently, CCT and CMRI have been proposed and tested as alternative imaging modalities. Given the noninvasive nature of CCT and CMR, developing strategies based on these modalities for the detection of cardiac thrombi and flow stasis is of great clinical interest. However, it must be shown that these advanced imaging techniques 
yield added value in the clinical setting.

\section{Conflicts of Interest}

The author declare that they have no conflict of interest.

\section{REFERENCES}

1. Go AS, Hylek EM, Phillips KA, Chang Y, Henault LE, Selby JV, et al. Prevalence of diagnosed atrial fibrillation in adults: national implications for rhythm management and stroke prevention: the AnTicoagulation and Risk Factors in Atrial Fibrillation (ATRIA) Study. JAMA 2001;285:23702375.

2. Han SW, Nam HS, Kim SH, Lee JY, Lee KY, Heo JH. Frequency and significance of cardiac sources of embolism in the TOAST classification. Cerebrovasc Dis 2007;24:463-468.

3. Bernhardt P, Schmidt H, Hammerstingl C, Lüderitz B, Omran H. Patients with atrial fibrillation and dense spontaneous echo contrast at high risk a prospective and serial follow-up over 12 months with transesophageal echocardiography and cerebral magnetic resonance imaging. J Am Coll Cardiol 2005;45:1807-1812.

4. Asinger RW, Koehler J, Pearce LA, Zabalgoitia M, Blackshear JL, Fenster $\mathrm{PE}$, et al. Pathophysiologic correlates of thromboembolism in nonvalvular atrial fibrillation: II. Dense spontaneous echocardiographic contrast (The Stroke Prevention in Atrial Fibrillation [SPAF-III] Study). J Am Soc chocardiogr 1999;12:1088-1096.

5. Fuster V, Rydén LE, Cannom DS, Crijns HJ, Curtis AB, Ellenbogen KA, et al. $2011 \mathrm{ACCF} / \mathrm{AHA} / \mathrm{HRS}$ focused updates incorporated into the ACC/ AHA/ESC 2006 Guidelines for the management of patients with atrial fibrillation: a report of the American College of Cardiology Foundation/ American Heart Association Task Force on Practice Guidelines developed in partnership with the European Society of Cardiology and in collaboration with the European Heart Rhythm Association and the Heart Rhythm Society. J Am Coll Cardiol 2011;57:e101-e198.

6. Manning WJ, Silverman DI, Gordon SP, Krumholz HM, Douglas PS. Cardioversion from atrial fibrillation without prolonged anticoagulation with use of transesophageal echocardiography to exclude the presence of atrial thrombi. N Engl J Med 1993;328:750-755.

7. Aschenberg W, Schlüter M, Kremer P, Schröder E, Siglow V, Bleifeld W. Transesophageal two-dimensional echocardiography for the detection of left atrial appendage thrombus. J Am Coll Cardiol 1986;7:163-166.

8. Shapiro MD, Neilan TG, Jassal DS, Samy B, Nasir K, Hoffmann U, et al. Multidetector computed tomography for the detection of left atrial appendage thrombus: a comparative study with transesophageal echocardiography. J Comput Assist Tomogr 2007;31:905-909.

9. Kim YY, Klein AL, Halliburton SS, Popovic ZB, Kuzmiak SA, Sola S, et al. Left atrial appendage filling defects identified by multidetector computed tomography in patients undergoing radiofrequency pulmonary vein antral isolation: a comparison with transesophageal echocardiography. Am Heart J 2007;154:1199-1205.

10. Hur J, Kim YJ, Nam JE, Choe KO, Choi EY, Shim CY, et al. Thrombus in the left atrial appendage in stroke patients: detection with cardiac CT angiography--a preliminary report. Radiology 2008;249:81-87.

11. Hur J, Kim YJ, Lee HJ, Ha JW, Heo JH, Choi EY, et al. Left atrial appendage thrombi in stroke patients: detection with two-phase cardiac CT angiography versus transesophageal echocardiography. Radiology 2009;251:683690.

12. Barkhausen J, Hunold P, Eggebrecht H, Schüler WO, Sabin GV, Erbel R, et al. Detection and characterization of intracardiac thrombi on MR imaging. AJR Am J Roentgenol 2002;179:1539-1544.

13. Mollet NR, Dymarkowski S, Volders W, Wathiong J, Herbots L, Rademakers FE, et al. Visualization of ventricular thrombi with contrast-enhanced magnetic resonance imaging in patients with ischemic heart disease. Circulation 2002;106:2873-2876.

14. Hur J, Kim YJ, Lee HJ, Nam JE, Hong YJ, Kim HY, et al. Cardioembolic stroke: dual-energy cardiac CT for differentiation of left atrial appendage thrombus and circulatory stasis. Radiology 2012;263:688-695.

15. Di Biase L, Santangeli P, Anselmino M, Mohanty P, Salvetti I, Gili S, et al. Does the left atrial appendage morphology correlate with the risk of stroke in patients with atrial fibrillation? Results from a multicenter study. J Am Coll Cardiol 2012;60:531-538.

16. Khurram IM, Dewire J, Mager M, Maqbool F, Zimmerman SL, Zipunnikov V, et al. Relationship between left atrial appendage morphology and stroke in patients with atrial fibrillation. Heart Rhythm 2013;10:18431849.

17. Pollick C, Taylor D. Assessment of left atrial appendage function by transesophageal echocardiography. Implications for the development of thrombus. Circulation 1991;84:223-231.

18. Shirani J, Alaeddini J. Structural remodeling of the left atrial appendage in patients with chronic non-valvular atrial fibrillation: implications for thrombus formation, systemic embolism, and assessment by transesophageal echocardiography. Cardiovasc Pathol 2000;9:95-101.

19. Ho SY, Cabrera JA, Sanchez-Quintana D. Left atrial anatomy revisited. Circ Arrhythm Electrophysiol 2012;5:220-228.

20. Agmon Y, Khandheria BK, Gentile F, Seward JB. Echocardiographic assessment of the left atrial appendage. J Am Coll Cardiol 1999;34:18671877.

21. Al-Saady NM, Obel OA, Camm AJ. Left atrial appendage: structure, function, and role in thromboembolism. Heart 1999;82:547-554.

22. Blackshear JL, Odell JA. Appendage obliteration to reduce stroke in cardiac surgical patients with atrial fibrillation. Ann Thorac Surg 1996;61: 755-759.

23. Nihoyannopoulos P, Smith GC, Maseri A, Foale RA. The natural history of left ventricular thrombus in myocardial infarction: a rationale in support of masterly inactivity. J Am Coll Cardiol 1989;14:903-911.

24. Asinger RW, Mikell FL, Elsperger J, Hodges M. Incidence of left-ventricular thrombosis after acute transmural myocardial infarction. Serial evaluation by two-dimensional echocardiography. N Engl J Med 1981;305:297302.

25. Stratton JR, Resnick AD. Increased embolic risk in patients with left ventricular thrombi. Circulation 1987;75:1004-1011.

26. Vaitkus PT, Barnathan ES. Embolic potential, prevention and management of mural thrombus complicating anterior myocardial infarction: a meta-analysis. J Am Coll Cardiol 1993;22:1004-1009.

27. Barbera S, Hillis LD. Echocardiographic recognition of left ventricular mural thrombus. Echocardiography 1999;16:289-295.

28. O'Gara PT, Kushner FG, Ascheim DD, Casey DE Jr, Chung MK, de Lemos JA, et al. 2013 ACCF/AHA guideline for the management of ST-elevation myocardial infarction: executive summary: a report of the American College of Cardiology Foundation/American Heart Association Task Force on Practice Guidelines. Circulation 2013;127:529-555.

29. Ogren M, Bergqvist D, Eriksson H, Lindblad B, Sternby NH. Prevalence and risk of pulmonary embolism in patients with intracardiac thrombosis: a population-based study of 23796 consecutive autopsies. Eur Heart J 2005;26:1108-1114

30. Chartier L, Béra J, Delomez M, Asseman P, Beregi JP, Bauchart JJ, et al. Free-floating thrombi in the right heart: diagnosis, management, and prognostic indexes in 38 consecutive patients. Circulation 1999;99:27792783.

31. Rose PS, Punjabi NM, Pearse DB. Treatment of right heart thromboemboli. Chest 2002;121:806-814.

32. Shrestha NK, Moreno FL, Narciso FV, Torres L, Calleja HB. Two-dimensional echocardiographic diagnosis of left-atrial thrombus in rheumatic heart disease. A clinicopathologic study. Circulation 1983;67:341-347.

33. Karakus G, Kodali V, Inamdar V, Nanda NC, Suwanjutah T, Pothineni KR. Comparative assessment of left atrial appendage by transesophageal and combined two- and three-dimensional transthoracic echocardiography. Echocardiography 2008;25:918-924.

34. Sallach JA, Puwanant S, Drinko JK, Jaffer S, Donal E, Thambidorai SK, et al. Comprehensive left atrial appendage optimization of thrombus using 
surface echocardiography: the CLOTS multicenter pilot trial. J Am Soc Echocardiogr 2009;22:1165-1172.

35. Hwang JJ, Chen JJ, Lin SC, Tseng YZ, Kuan P, Lien WP, et al. Diagnostic accuracy of transesophageal echocardiography for detecting left atrial thrombi in patients with rheumatic heart disease having undergone mitral valve operations. Am J Cardiol 1993;72:677-681.

36. Manning WJ, Weintraub RM, Waksmonski CA, Haering JM, Rooney PS, Maslow AD, et al. Accuracy of transesophageal echocardiography for identifying left atrial thrombi. A prospective, intraoperative study. Ann Intern Med 1995;123:817-822.

37. Transesophageal echocardiographic correlates of thromboembolism in high-risk patients with nonvalvular atrial fibrillation. The stroke prevention in Atrial Fibrillation Investigators Committee on Echocardiography. Ann Intern Med 1998;128:639-647.

38. Nakajima H, Seo Y, Ishizu T, Yamamoto M, Machino T, Harimura Y, et al. Analysis of the left atrial appendage by three-dimensional transesophageal echocardiography. Am J Cardiol 2010;106:885-892.

39. Blendea D, Heist EK, Danik SB, Barrett C, Ruskin JN, Mansour M. Analysis of the left atrial appendage morphology by intracardiac echocardiography in patients with atrial fibrillation. J Interv Card Electrophysiol 2011:31:191-196.

40. Ren JF, Marchlinski FE, Supple GE, Hutchinson MD, Garcia FC, Riley MP, et al. Intracardiac echocardiographic diagnosis of thrombus formation in the left atrial appendage: a complementary role to transesophageal echocardiography. Echocardiography 2013;30:72-80.

41. Saksena S, Sra J, Jordaens L, Kusumoto F, Knight B, Natale A, et al. A prospective comparison of cardiac imaging using intracardiac echocardiography with transesophageal echocardiography in patients with atrial fibrillation: the intracardiac echocardiography guided cardioversion helps interventional procedures study. Circ Arrhythm Electrophysiol 2010;3: 571-577.

42. American College of Cardiology Foundation Appropriate Use Criteria Task Force; American Society of Echocardiography; American Heart Association; American Society of Nuclear Cardiology; Heart Failure Society of America; Heart Rhythm Society, et al. ACCF/ASE/AHA/ASNC/HFSA/ HRS/SCAI/SCCM/SCCT/SCMR 2011 Appropriate Use Criteria for Echocardiography. A Report of the American College of Cardiology Foundation Appropriate Use Criteria Task Force, American Society of Echocardiography, American Heart Association, American Society of Nuclear Cardiology, Heart Failure Society of America, Heart Rhythm Society, Society for Cardiovascular Angiography and Interventions, Society of Critical Care Medicine, Society of Cardiovascular Computed Tomography, Society for Cardiovascular Magnetic Resonance American College of Chest Physicians. J Am Soc Echocardiogr 2011;24:229-267.

43. Stratton JR, Lighty GW Jr, Pearlman AS, Ritchie JL. Detection of left ventricular thrombus by two-dimensional echocardiography: sensitivity, specificity, and causes of uncertainty. Circulation 1982;66:156-166.

44. Visser CA, Kan G, David GK, Lie KI, Durrer D. Two dimensional echocardiography in the diagnosis of left ventricular thrombus. A prospective study of 67 patients with anatomic validation. Chest 1983;83:228-232.

45. Chen C, Koschyk D, Hamm C, Sievers B, Kupper W, Bleifeld W. Usefulness of transesophageal echocardiography in identifying small left ventricular apical thrombus. J Am Coll Cardiol 1993;21:208-215.

46. Pearson AC. Transthoracic echocardiography versus transesophageal echocardiography in detecting cardiac sources of embolism. Echocardiography 1993;10:397-403.

47. Thanigaraj S, Schechtman KB, Pérez JE. Improved echocardiographic delineation of left ventricular thrombus with the use of intravenous secondgeneration contrast image enhancement. J Am Soc Echocardiogr 1999;12: 1022-1026.

48. Swinburn J, Lahiri A, Senior R. Tissue harmonic imaging: a new method for predicting left ventricular thrombus? J Am Soc Echocardiogr 2000;13: 680-681.

49. Schwartzbard AZ, Tunick PA, Rosenzweig BP, Kronzon I. The role of transesophageal echocardiography in the diagnosis and treatment of right atrial thrombi. J Am Soc Echocardiogr 1999;12:64-69.

50. Achenbach S, Sacher D, Ropers D, Pohle K, Nixdorff U, Hoffmann U, et al. Electron beam computed tomography for the detection of left atrial thrombi in patients with atrial fibrillation. Heart 2004;90:1477-1478.

51. Singh NK, Nallamothu N, Zuck VP, Issa ZF. Left atrial appendage filling defects on 64-slice multidetector computed tomography in patients undergoing pulmonary vein isolation: predictors and comparison to transesophageal echocardiography. J Comput Assist Tomogr 2009;33:946-951.

52. Romero J, Husain SA, Kelesidis I, Sanz J, Medina HM, Garcia MJ. Detection of left atrial appendage thrombus by cardiac computed tomography in patients with atrial fibrillation: a meta-analysis. Circ Cardiovasc Imaging 2013;6:185-194.

53. Kim SC, Chun EJ, Choi SI, Lee SJ, Chang HJ, Han MK, et al. Differentiation between spontaneous echocardiographic contrast and left atrial appendage thrombus in patients with suspected embolic stroke using twophase multidetector computed tomography. Am J Cardiol 2010;106:11741181.

54. Hur J, Kim YJ, Lee HJ, Nam JE, Ha JW, Heo JH, et al. Dual-enhanced cardiac CT for detection of left atrial appendage thrombus in patients with stroke: a prospective comparison study with transesophageal echocardiography. Stroke 2011;42:2471-2477.

55. Budoff MJ. Maximizing dose reductions with cardiac CT. Int J Cardiovasc Imaging 2009;25:279-287.

56. Patino M, Prochowski A, Agrawal MD, Simeone FJ, Gupta R, Hahn PF, et al. Material separation using dual-energy CT: current and emerging applications. Radiographics 2016;36:1087-1105.

57. Scheske JA, O’Brien JM, Earls JP, Min JK, LaBounty TM, Cury RC, et al. Coronary artery imaging with single-source rapid kilovolt peak-switching dual-energy CT. Radiology 2013;268:702-709.

58. Johnson TR, Krauss B, Sedlmair M, Grasruck M, Bruder H, Morhard D, et al. Material differentiation by dual energy CT: initial experience. Eur Radiol 2007;17:1510-1517.

59. Hong YJ, Hur J, Kim YJ, Lee HJ, Hong SR, Suh YJ, et al. Dual-energy cardiac computed tomography for differentiating cardiac myxoma from thrombus. Int J Cardiovasc Imaging 2014;30 Suppl 2:121-128.

60. Koć M, Kostrubiec M, Elikowski W, Meneveau N, Lankeit M, Grifoni S, et al. Outcome of patients with right heart thrombi: the Right Heart Thrombi European Registry. Eur Respir J 2016;47:869-875.

61. Torbicki A, Galié N, Covezzoli A, Rossi E, De Rosa M, Goldhaber SZ; ICOPER Study Group. Right heart thrombi in pulmonary embolism: results from the International Cooperative Pulmonary Embolism Registry. J Am Coll Cardiol 2003;41:2245-2251.

62. Rustemli A, Bhatti TK, Wolff SD. Evaluating cardiac sources of embolic stroke with MRI. Echocardiography 2007;24:301-308; discussion 308.

63. Rathi VK, Reddy ST, Anreddy S, Belden W, Yamrozik JA, Williams RB, et al. Contrast-enhanced CMR is equally effective as TEE in the evaluation of left atrial appendage thrombus in patients with atrial fibrillation undergoing pulmonary vein isolation procedure. Heart Rhythm 2013;10:10211027.

64. Srichai MB, Junor C, Rodriguez LL, Stillman AE, Grimm RA, Lieber ML, et al. Clinical, imaging, and pathological characteristics of left ventricular thrombus: a comparison of contrast-enhanced magnetic resonance imaging, transthoracic echocardiography, and transesophageal echocardiography with surgical or pathological validation. Am Heart J 2006;152:75-84.

65. Weinsaft JW, Kim HW, Shah DJ, Klem I, Crowley AL, Brosnan R, et al. Detection of left ventricular thrombus by delayed-enhancement cardiovascular magnetic resonance prevalence and markers in patients with systolic dysfunction. J Am Coll Cardiol 2008;52:148-157.

66. Weinsaft JW, Kim J, Medicherla CB, Ma CL, Codella NC, Kukar N, et al. Echocardiographic algorithm for post-myocardial infarction LV thrombus: a gatekeeper for thrombus rvaluation by delayed enhancement CMR. JACC Cardiovasc Imaging 2016;9:505-515.

67. Roifman I, Connelly KA, Wright GA, Wijeysundera HC. Echocardiography vs. cardiac magnetic resonance imaging for the diagnosis of left ventricular thrombus: a systematic review. Can J Cardiol 2015;31:785-791. 
68. Kitkungvan D, Nabi F, Ghosn MG, Dave AS, Quinones M, Zoghbi WA, et al. Detection of LA and LAA thrombus by CMR in patients referred for pulmonary vein isolation. JACC Cardiovasc Imaging 2016;9:809-818.

69. Rodgers CT, Robson MD. Cardiovascular magnetic resonance: physics and terminology. Prog Cardiovasc Dis 2011;54:181-190.

70. Dass S, Suttie JJ, Piechnik SK, Ferreira VM, Holloway CJ, Banerjee R, et al. Myocardial tissue characterization using magnetic resonance noncontrast t1 mapping in hypertrophic and dilated cardiomyopathy. Circ Cardiovasc Imaging 2012;5:726-733.

71. Hamlin SA, Henry TS, Little BP, Lerakis S, Stillman AE. Mapping the future of cardiac MR imaging: case-based review of T1 and T2 mapping techniques. Radiographics 2014;34:1594-1611.

72. Kim PK, Hong YJ, Im DJ, Suh YJ, Park CH, Kim JY, et al. Myocardial T1 and T2 mapping: techniques and clinical applications. Korean J Radiol 2017;18:113-131.

73. Pazos-López P, Pozo E, Siqueira ME, García-Lunar I, Cham M, Jacobi A, et al. Value of CMR for the differential diagnosis of cardiac masses. JACC Cardiovasc Imaging 2014;7:896-905.

74. Caspar T, El Ghannudi S, Ohana M, Labani A, Lawson A, Ohlmann P, et al. Magnetic resonance evaluation of cardiac thrombi and masses by T1 and T2 mapping: an observational study. Int J Cardiovasc Imaging 2017; 33:551-559. 